Michel COTTE, Le choix de la révolution industrielle. Les entreprises de Marc Seguin et ses frères (1815-1835)

François Jarrige

URL : http://journals.openedition.org/rh19/3539

DOI : $10.4000 /$ rh19.3539

ISSN : $1777-5329$

Éditeur

La Société de 1848

Édition imprimée

Date de publication : 15 novembre 2008

Pagination : 185-242

ISSN : 1265-1354

Référence électronique

François Jarrige, "Michel cotTe, Le choix de la révolution industrielle. Les entreprises de Marc Seguin et ses frères (1815-1835) », Revue d'histoire du XIXe siècle [En ligne], 37 | 2008, mis en ligne le 01 décembre 2008, consulté le 22 septembre 2020. URL : http://journals.openedition.org/rh19/3539; DOI : https:// doi.org/10.4000/rh19.3539

Ce document a été généré automatiquement le 22 septembre 2020.

Tous droits réservés 


\title{
Michel coTTE, Le choix de la révolution industrielle. Les entreprises de Marc Seguin et ses frères (1815-1835)
}

\author{
François Jarrige
}

\section{RÉFÉRENCE}

Michel сОтте,Le choix de la révolution industrielle. Les entreprises de Marc Seguin et ses frères (1815-1835), Rennes, Presses universitaires de Rennes, collection Carnot, 2007, 572 p. ISBN : 975-2-7535-0476-9. 24 euros.

1 Selon Michel Cotte, «plonger dans l'aventure des frères Seguin, de leur aîné Marc tout particulièrement ", c'est "faire un saut directement au cœur de la création d'un ensemble d'entreprises [...] qui vont accompagner et façonner l'industrialisation de la France au début du XIX siècle» (p. 9). Arrivé au terme de ce gros ouvrage qui conclut de nombreuses années de recherches minutieuses dans des fonds d'archives en partie inédits ${ }^{1}$, on ne peut qu'être d'accord avec le constat et reconnaître le rôle important joué par les Seguin dans l'industrialisation de la France et, au-delà, de l'Europe. Pour comprendre l'action de cette famille, Michel Cotte ne propose pas simplement une biographie d'entrepreneurs, il reconstitue aussi les étapes successives et les contextes économiques et techniques qui ont rendu possibles leurs innovations industrielles.

2 Suivant une démarche chrono-thématique, l'auteur analyse d'abord les racines de la réussite économique des Seguin. La famille s'était installée à Annonay dans le négoce des draps au XVIII ${ }^{\mathrm{e}}$ siècle. À la fin de l'Empire, les Seguin se tournent vers la production. Sous la Restauration, ils diversifient peu à peu leur activité en mécanisant le travail et en étendant la gamme de leurs produits et de leurs marchés. Même si la draperie traditionnelle piétine sous la Restauration, les Seguin s'enrichissent peu à peu, et c'est cette activité qui leur fournit les capitaux pour investir ensuite dans d'autres opérations industrielles. Marc Seguin, l'aîné de la famille, bénéficie à la fois d'une 
formation scientifique et technique poussée et d'un environnement économique régional dynamique qui favorise les alliances fructueuses (la mère des frères Seguin était ainsi la nièce des inventeurs du ballon à air chaud, les papetiers Étienne et Joseph de Montgolfier). À partir de 1825, la draperie passe progressivement au second plan de leurs affaires au profit des travaux publics et des transports qui vont constituer l'épine dorsale de leur activité. En tant qu'historien des techniques, Michel Cotte privilégie l'analyse des innovations techniques et son étude est construite comme une typologie des bouleversements technologiques successifs introduits par les Seguin.

Le redéploiement de leurs activités commence avec la construction des ponts suspendus dont les Seguin deviennent rapidement des spécialistes reconnus (chapitres IV-X). Le projet des frères Seguin de construction d'un pont suspendu sur le Rhône apparaît durant l'hiver 1821-1822, ils vont réussir à appliquer cette technique déjà utilisée par les Américains et les Anglais en l'adaptant à la situation géographique rhodanienne et aux singularités économiques locales. Dans les années 1820 les installations anciennes deviennent autant de goulots d'étranglement pour la croissance économique régionale, les Seguin vont jouer de ces contraintes pour obtenir de l'État les concessions indispensables et imposer leurs solutions techniques. Mais en dépit de leur réussite, les Seguin semblent se détourner assez rapidement des constructions de ponts suspendus, laissant à d'autres le soin de tirer tous les profits de cette technique nouvelle à la fin des années 1820. Selon Michel Cotte, ce paradoxe s'explique par l'attitude " pragmatique et lucide » de Marc Seguin, «capable de remettre en cause ses propres innovations en fonction de circonstances nouvelles!» (p. 234). À cette date en effet, le standard Seguin du pont suspendu léger apparaît peu adapté au chemin de fer en pleine expansion.

4 Avant d'analyser en détail l'action des Seguin dans le secteur ferroviaire, l'auteur s'arrête longuement sur leur action dans le développement de la navigation à vapeur sur le Rhône (chapitres XI-XV). Les premiers projets de mécanisation des transports fluviaux remontent à la fin du XVIII ${ }^{e}$ siècle, des idées techniques originales mais sans lendemain sont formulées comme le bateau aquamoteur qui cherchait à utiliser le courant des rivières comme source d'énergie utilisable pour le mouvement de remonte. Mais c'est la nouvelle force motrice de la vapeur qui va permettre de réaliser certaines conceptions utopiques des Lumières autour de 1820. Là encore, les expériences américaines et anglaises jouent un rôle décisif dans les projets des Seguin. En 1825, Marc Seguin crée avec plusieurs associés une société du halage à la vapeur sur le Rhône, parallèlement il passe commande de machines à vapeur haute pression auprès d'un constructeur londonien. Les premières expériences de navigation ont lieu durant l'hiver 1826-1827 au terme d'intenses débats et discussions techniques. Avec un sens affûté de la communication, les Seguin orchestrent une campagne d'opinion (articles dans la presse locale et nationale, chansons propagées dans les rues de Lyon) pour vanter le succès de ces essais de navigation. Mais la réalité technique est plus cruelle : la machine à vapeur est défectueuse et chaque essai nécessite de coûteuses réparations. Malgré cela, la navigation commerciale commence en 1827. Mais les difficultés commerciales liées aux accidents et les incertitudes techniques ont finalement raison de l'entreprise des Seguin qui se détournent peu à peu de la navigation à vapeur. Il faut dire qu'entre temps, le chemin de fer était devenu la grande affaire à laquelle ils allaient se consacrer entièrement. 
5 Le reste de l'ouvrage (chapitres XVI-XXII) est consacré au rôle des Seguin dans le développement de la première ligne de chemin de fer entre Saint-Étienne et Lyon au tournant des années 1830. Le 27 mars 1826, les frères Seguin et Édouard Biot obtiennent l'adjudication de la ligne de chemin de fer de Saint-Étienne à Lyon. Afin de réaliser la jonction de la Loire au Rhône le chemin de fer passe dans la vallée accidentée du Gier : par Saint-Chamond, Rive-de-Gier et Givors, sur une distance de 58 kilomètres. La "Compagnie du chemin de fer de Saint-Étienne à Lyon" dut acheter quelques neuf cents parcelles de terrain, nécessaires pour la réalisation de la ligne. Ces acquisitions mènent, pour la plupart, à de coûteuses tractations. Mais il était indispensable que le tracé soit le plus commode possible pour permettre l'utilisation du chemin de fer. Le tracé selon Seguin devait corriger la nature, il comportait un pont sur la Saône, un viaduc, des ponts et quatorze souterrains, dont celui de Terrenoire qui mesurait 1500 mètres. Le coût total de la construction de la ligne s'élève à la somme considérable de plus de 15 millions de francs, faisant de la Compagnie l'une des plus importantes sociétés du pays. Marc Seguin devait aussi se procurer les machines locomotives qu'il avait pu voir fonctionner lors de son voyage en Angleterre à la fin de l'année 1825. En mars et avril 1828, il achète aux ateliers Stephenson de Newcastle deux locomotives « Locomotion » d'occasion. Seguin construit douze machines dotées de son propre modèle de chaudière tubulaire et de tirage forcé, il sextuple ainsi la production de vapeur et augmente la vitesse maximum de la machine initiale. La première locomotive de Marc Seguin est mise en service d'une manière concluante lors d'essais en novembre et décembre 1829. Une deuxième machine est achevée en juin 1830, après la prise d'un brevet. On voit que Marc Seguin est parvenu à perfectionner la machine de Stephenson en l'associant au principe de la "chaudière tubulaire " qu'il avait mis au point et qui permettait de décupler la surface de chauffe en faisant passer dans des tubes l'air brûlant issu du foyer.

6 Finalement, l'ouverture de la ligne a lieu au début de l'été 1830, juste avant le déclenchement des événements révolutionnaires. L'étude des débuts de l'exploitation ferroviaire est l'occasion d'une intéressante analyse des répercussions de la crise révolutionnaire sur l'entreprise des Seguin. Profitant du contexte d'agitation sociale, les ouvriers crocheteurs, auparavant employés au déchargement manuel de la houille, et privés de travail par l'installation d'une trappe au fond du wagon, se révoltent contre les installations et contraignent les Seguin à négocier. Au terme de multiples difficultés, sociales et commerciales, l'ensemble de l'exploitation ouvre finalement en février 1833. Pour rentabiliser l'affaire, les Seguin doivent rapidement réaliser un trafic maximum : ils y parviennent et commencent à engendrer des bénéfices. Mais c'est à ce moment que Marc Seguin, en conflit de plus en plus ouvert avec le conseil d'administration et les actionnaires de la compagnie qui s'opposent à ses projets jugés trop coûteux, dépose sa démission. Ce conflit peut s'interpréter, selon Michel Cotte, par le refus de la haute banque de partager son pouvoir avec les entrepreneurs. Face aux financiers avides, Marc Seguin incarnerait donc l'entrepreneur courageux et ingénieux. On aimerait néanmoins en savoir un peu plus sur cette décision et ses motivations qui sont examinées rapidement.

7 En 1838, Marc Seguin s'installe finalement dans l'abbaye cistercienne désaffectée de Fontenay dans la Côte-d'Or, qui avait été achetée en 1820 par son parent Élie de Montgolfier. Là, il vit comme un sage entouré de sa nombreuse famille, développant plusieurs projets scientifiques et techniques. Se détournant du terrain de l'industrie, il 
se consacre aux recherches scientifiques avec l'espoir - largement déçu-d'être pleinement reconnu par la communauté scientifique. En 1839, il publie son ouvrage De l'influence des chemins de fer et de l'art de les construire et de les tracer; dans les années 1840 il se passionne pour le chemin de fer atmosphérique puis, dans les années 1850, il élabore un prototype de nouveau moteur baptisé machine pulmonaire qui ne donnera lieu à aucune application directe. Il meurt en 1875 après s'être intéressé à l'aviation. Mais cette deuxième moitié de la vie de Marc Seguin, marquée par l'aisance et le retrait des affaires après 1835, intéresse peu Michel Cotte qui s'était fixé comme objectif d'étudier les entreprises des Seguin.

Qu'il s'agisse des ponts suspendus, des bateaux à vapeur, des chemins de fer ou de la construction des machines locomotives, les entreprises des Seguin forment en définitive un ensemble complémentaire d'entreprises de génie civil et de transport en région lyonnaise. Ils ont introduit un bouleversement des transports, inspiré par l'exemple britannique mais adapté à des conditions territoriales et humaines singulières. L'une des forces de l'ouvrage de Michel Cotte est qu'il ne se concentre pas uniquement sur les destins individuels mais qu'il a le souci permanent de les réinscrire dans les contextes techniques, géographiques et culturels plus vastes ainsi que dans les réseaux locaux et internationaux d'interconnaissance sur lesquels la famille appuyait son dynamisme. Grâce à cela, il évite de tomber dans le défaut traditionnel de l'histoire des techniques facilement portée à l'hagiographie. L'étude d'une famille industrielle permet par ailleurs de mettre en lumière les logiques qui permettent le passage d'une branche à l'autre et les processus par lesquels les innovations dans un secteur sont retraduites dans un autre. Il aurait néanmoins pu être intéressant de tenter une approche comparée avec d'autres figures industrielles connues de l'époque, comme Neuflize à Sedan, Dufaud le créateur de Fourchambault, ou encore les industriels mulhousiens engagés à l'époque dans la mécanisation.

9 Fasciné par les Seguin dont il s'efforce de comprendre « la réussite » et le rôle dans le « décollage » (p.525) économique français, Michel Cotte n'accorde en revanche guère de place aux enjeux historiographiques et méthodologiques que soulève son étude et qu'il aborde rapidement en conclusion. S'inscrivant pleinement dans le champ de l'histoire des techniques, il évacue en quelques lignes les apports « de l'économie et de la sociologie en particulier", considérant, trop rapidement à notre avis, que "ces propositions méthodologiques semblent surtout devoir s'appliquer aux tentatives de synthèse, aux explications de caractère global, soit sur la longue durée de l'histoire des techniques ou sur de larges panoramas d'une société industrielle donnée » (p.517). L'ouvrage de Michel Cotte reste traditionnel dans son approche, il accorde toujours la première place à l'entrepreneur innovant, il maintient le modèle général d'une "révolution industrielle», notant simplement que ce "terme fut parfois remis en cause ", et celui d'une supériorité britannique à l'aune de laquelle on peut évaluer l'action des Seguin. Une analyse plus réflexive de la notion de "révolution industrielle » qui n'existait pas lorsque les Seguin ont entamé leur croissance aurait permis, sans doute, d'éviter davantage le piège du regard rétrospectif et du déterminisme technologique au profit d'une réflexion sur les voies plurielles et singulières de l'industrialisation européenne. Une plus grande attention à la mythologie familiale et à ses fonctions, à la construction ultérieure de Marc Seguin comme exemple typique de la figure de l'industriel entreprenant aurait également été intéressante. Par ailleurs, l'ouvrage n'aurait sans doute rien perdu à être parfois plus synthétique et bref, mais les spécialistes seront sans doute heureux d'y trouver tous les 
menus détails techniques que l'auteur maîtrise admirablement bien. Chacun des chapitres du livre peut d'ailleurs se lire aussi comme l'étude d'une technique particulière ou d'une étape décisive de l'évolution des frères Seguin. Comme le montre finalement bien Michel Cotte, la force des Seguin a résidé dans leur capacité à articuler les théories des techniciens et le champ des possibles qu'offrait le système productif de leur époque. À ce titre, ce livre constitue un apport important à la compréhension de la première industrialisation.

\section{NOTES}

1. . Une thèse de doctorat est à l'origine de ce livre: Michel Cotte, Innovation et transfert de technologies: le cas des entreprises de Marc Seguin, France, 1815-1835, Thèse d'histoire, sous la direction de Louis Bergeron, EHESSS, 1992, $1142 \mathrm{f}^{\circ}$; l'auteur a également édité un utile recensement du fonds d'archives Seguin : Michel Cotte, Le fonds d'archives Seguin, aux origines de la révolution industrielle, 1790-1860, Privas, Éditions des archives départementales de l'Ardèche, 1997. 